\section{SAN MICHELE. ENTRE CIELO Y MAR}

SAN MICHELE, BETWEEN SKY AND SEA

Pablo Blázquez Jesús

RSUMEN El cementerio es uno de los tipos arquitectónicos más profundos y metafóricos. El concurso para la ampliación del

so para la ampliación del de pruebas sobre el que poder analizar el contexto historicico en torno a estata tipología, y yu relación con la ciudad y yl territorio. El estudio de este caso concreto nos permite descubrir personajes, relaciones casuales y hallazgos que se despliegan a lo largo del lexto. La historia del cementerio de San Michele es tambien la cronica de la transtormación de la ciudad de Venecia y su Laguna.

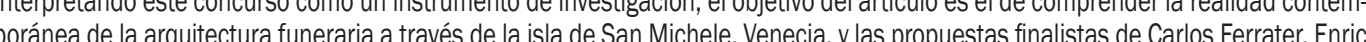
Miralles y David Chipperfield. Una historia bajo la cual se vislumbran claves que nos sirven para reflexionar acerca del cementerio

PALABRAS CLAVE cementerio; Venecia; concurso; Ferrater; Miralles; Chipperfield.

SUMMARY The cemetery is one of the most profound and metaphorical kinds of architecture. The competition for the extension cemetery called in 1998 by the Venice analyse the historical context surrounding this type of architecture, and its relationship with the city and the region. The study of this particular case allows us to uncover characters, casual relationships and findingss that unfold throughout the text. The history of the San Michele cemetery is also the chronicle of the transformation of the city of Venice and its Lagoon. Interpreting this competition as a research tool, the aim or the paper is to understand he contemporary reality of funerary architecture through the island of San glimpsed that serve our reflection about the contemporary cemetery, the city and the region.

WORDS cemetery; Venice; competition; Ferrater; Miralles; Chipperff

r.com. Arquitecto: BSO arquitectura

\section{ANTECEDENTES}

E I cementerio como tipo arquitectónico por ancla en la antigüedad del hombre, nos proyecta ( desde el pasado hacia el futuro, no existe practicamente cullura alguna que abandone a sus difuntos sin ceremotias o monumentos en su memoria. Nichos en allura. tumbas, lápicas, panteones, columbarios, túmulos... son mas las construciones funerarias resullado de una gur glenes efen ydes e historias de personas que habitaron un predum a lo las lanstomaciones que se han producido a lo largo del tiempo en la sociedades, simbolos de una evolucion en laconciencia del ser humano. manes entendidos como fionteras de conlacto entre el mundo de los vivos y el de los muertos desde los que percibir el peso de nuestros antepasados y comprender Porizonte de nuestra existencia.

La historla de los recintos funerarios está repleta de oscliaciones pendulares en el tiempo. Desde el siglo a.C. las inhumaciones se realizaron en las iglesias o en el interior de sus dependencias consagradas, mientras en los patios contiguos al templo religioso en los que se abrian grandes fosas. Conventos y hospitales tambien aproxmactanente el siglo XVVI. A comienzos de la Edad Contemporánea, las cludades se habian convertido en grandes cementerios que con el avance del siglo XVIII. hacian insostenible los problemas de espacio intramuros. La colmalación de los camposantos junto a las denuncias de insalubridad por estas prácticas comenzaron a tener un importante protagonismo en la sociedad que empezaba a abrazar los ideales de la llustración. Las grandes epidemias que azolaban Europa supusieron un punto de inflexión para los cementerios que se convirtieron en simples infraestructuras de servicio condenados a un extio necesario que ordenaba la construcción de nuevos recintos funcralos alrededor de las ciudades europeas. El siglo XIX trajo consigo la planificación de autenticas ciudades para los muertos, lugares alejados de los angustiosos emplazamientos urbanizados, cerrados, introvertidos y mono-funcionales que se reflejan por ejemplo Durocipolis parisina de Pere-Lachaise.

Durante el siglo XX las necrópolis siguieron vinculados a conceptos decimonónicos. El aumento de la población debido a la continua expansion urbana y las dos guerras mindialas provocaron numerosos problemas de 


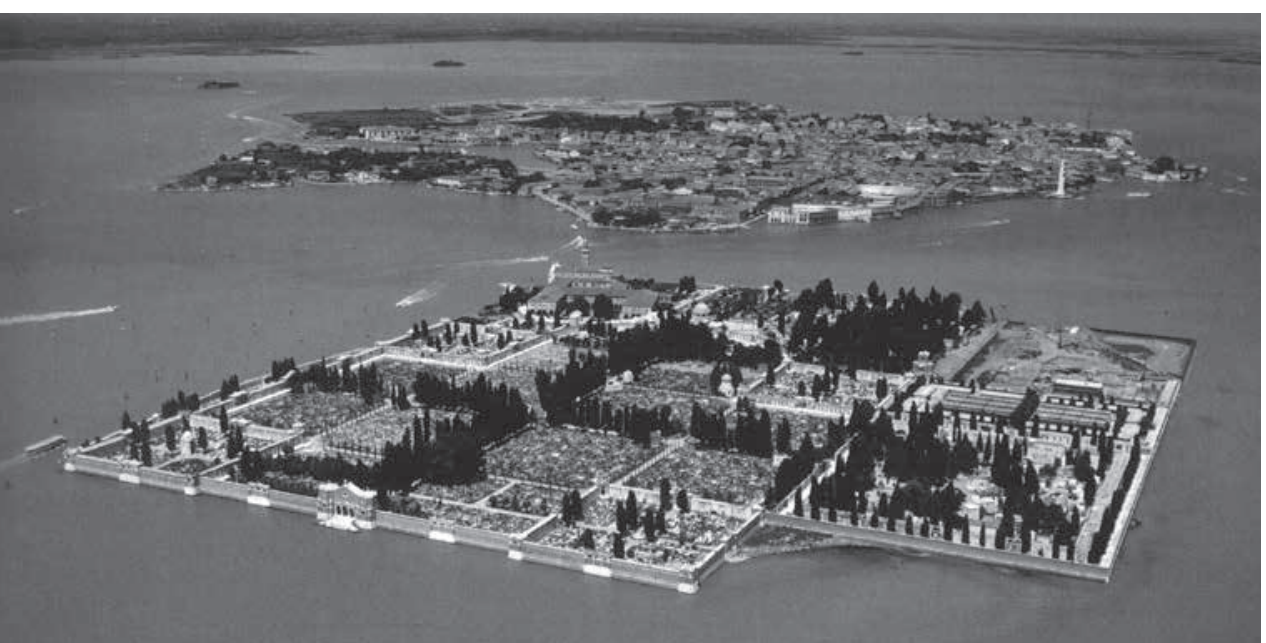

1. Islas de San Michele y Murano 2. Bordone, Benedetto:" "LagunadeVenecia". 3. Isla de Sacca Matia adoptó la solución del crecimiento en altura mediante "nichos". Los cementerios intentaron aprovechar al máximo el espacio disponible extendiendo esta solución hasta la saciedad, como si de un aparcamiento para difuntos se Hitos aislados, como el Cementerio Ideal de Teodoro Anasagasti, o los Monumentos y Memoriales a los caídos en las guerras del siglo XX, hacia el que mostraron especial interés los arquitectos del Movimiento Moderno, no contribuyeron por si solos a aliviar la sensación de abandono de la arquitectura funeraria. Algunas obras provocaron un impacto inicial como el concurso convocado en el año 1915 para el cementerio de Estocolmo y posteriormente construido por Asplund y Lewerentz (primer acercamiento del Movimiento Moderno a esta tipología arquitectónica), la ampliación en 1971 de San Cataldo en Módena de Aldo Rossi, o el dialogo entre tumba y cementerio planeado por Carlo Scarpa en Allivole para la familia Brion inalizado en el año 1978. Ejemplos que nos hicieron preguntarnos si la arquitectura funeraria se encontraba en condiciones de vivir una nueva revisión histórica.

Alcanzamos aquí un punto de inflexión en este breve recorrido histórico. A partir de los años cincuenta del siglo $X X$, el cementerio no se plantea en modo alguno como materia de discusion. Los arquitectos contemporaneaos se han centrado en el desarrollo y la investigación de otras tipologias arquitectónicas alejadas de la tipologia funeraria, sin embargo algunos proyectos han alcanzado en los últimos tiempos una importante trascendencia, no exenta de criticas y rechazos por parte de la sociedad como los ejemplos de Finisterra de Cesar Portela, Igualada de Enric Miralles y Carme Pinos, o fuera de nuestras Ironteras el Sky Cemetery de NL Architects que plantean a posibilidad de construir una necropolis a modo de rascacielos en el centro de la ciudad de Nueva York.
ISLA, PAISAJE Y TERRITORIO

San Michele asoma entre Venecia y Murano cerrada sobre si misma. Un pedazo de tierra suspendido entre cielo (figura 1). Un camposanto conocido bajo el sobrenombre de "la isla de los muertos" y en el que descansan personajes ilustres como Igor Stravinsky, Luchino Visconti o Joseph Brodsky

El concurso convocado en el año 1998 por la Administración Municipal de Venecia propone la necesaria, pero a la vez compleja, ampliacion de su cementerio. La ectura de la intervención debe de ser observada a través de una mirada lejana, estirando la sabana del pensamiento arquitectónico sobre la realidad detallada, descubriendo un nuevo territorio transformado, sin alterarlo. Miradas que desvelarán nuevas situaciones, personajes e historias escondidas bajo cada una de las intervenciones presentadas.

La Laguna de Venecia con $550 \mathrm{~km}^{2}$ y más de $50 \mathrm{~km}$ de límites de arena y tierra, es la pieza clave para comprender las transtomaciones y acontecimientos que han erminado por allerar de forma radical la realidad de este lugar. Un paisaje marcado por su horizontalidad, bañado por una gran superficie de agua que sollo se abre en tres puertos hacia el Mediteráneo, desde donde entra el agua salada y se expulsa salobre gracias al movimiento de las mareas. Durante los años de la República Veneciana, la Laguna estaba formada por un archipielelago de islas elevadas sobre el mar, siendo Venecia su epicentro, cada una de ellas con una función espectica, muy ligada a su situación y caracteristicas (figura 2). Un paisaje que con el paso del tiempo se ha ido transtormando y bajo el cua descansan antiguas islas como Centranica, Ammiana y conectaban las islas que formaban parte de este sistema

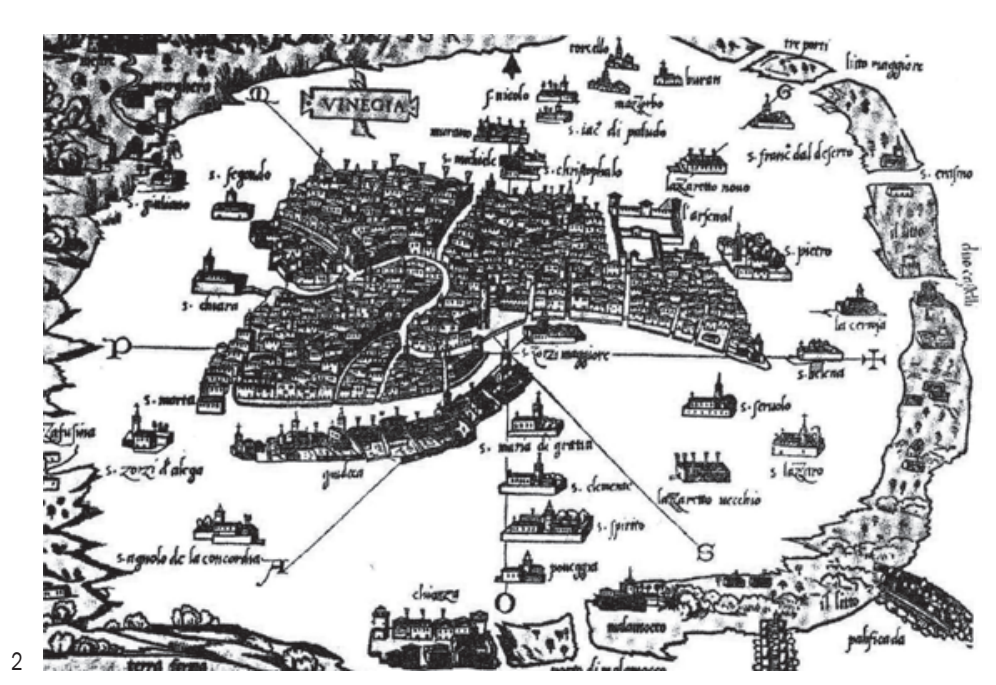

la Laguna funcionaba como un espacio de tránsito, intersticial. Todavía hoy se pueden observar grupos de pilotes que emergen sobre el agua marcando el recorrido que deben seguir las embarcaciones para evitar bajios, bancos de arena o algunas de estas islas hundidas. El progresivo descenso en el comercio debido a la glo XX, y el imparable desarrollo industrial, reflejaron la incapacidad de crecimiento comercial que sufría Venecia dado su aislamiento. La iniciativa de construir un puente que conectara Venecia con tierra firme en el año 1931 generó tal transformación sobre la isla y el sistema, que a Laguna pasó de ser el único medio de conexión entre as islas, a ser entendida como un obstaculo. La actividad económica se centró entre Venecia y Murano, quedando prácticamente olvidados el resto de pequeños islotes. Todo aquel archipiélago de islas interdependientes se desvanecio entonces dando paso a esta "otra Venecia" que se bate entre dos mareas, la de la propia naturaleza que amenaza constantemente la construcción de la

ciudad, y la de los turistas ávidos de recorrer sus calles comercios

Aquella obra modificó radicalmente la concepción de Venecia como pieza fundamental del sistema lagunar. Sin embargo Anne Lacaton, arquitecta y miembro del juracurso del Parque de la Laguna de Venecia realzado en el 2008 escribia lo siguiente:" Viajar a Venecia sim pasar por San Marcos, surcando la Laguna en una pequena ombarcación, de isla en isla, los ojos a ras de aqua La una extrana relación con el paisaje y el territorio Las palabras de Anne Lacaton muestran la existencia aún hoy de la antigua Venecia, lejos de ese mundo de canales, puentes y mascaras carnavalescas. Un lugar más, dentro del conjunto de islas olvidadas que aún siguen Es Es este envalor que subyace oculto tras el concurso para la amplación del cementerio. El verdadero interés que dia de hoy la isla de San Michele es e de seguir siendo una isla, que se protege del envite de las mareas cerrándose sobre sí misma, a la espera de la 


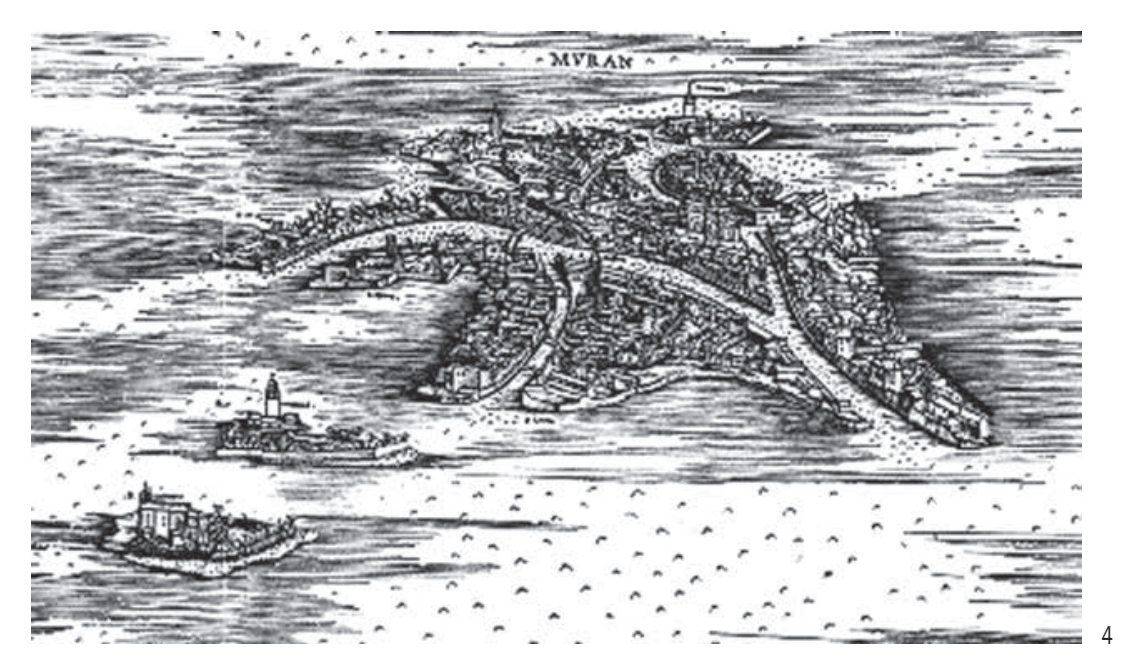

4. "Illa de San Michele y San Cristofóor della Pace

(5. Backlin Arnold: "La isla de los muertos".

6. Fases para la ampliación del concurso

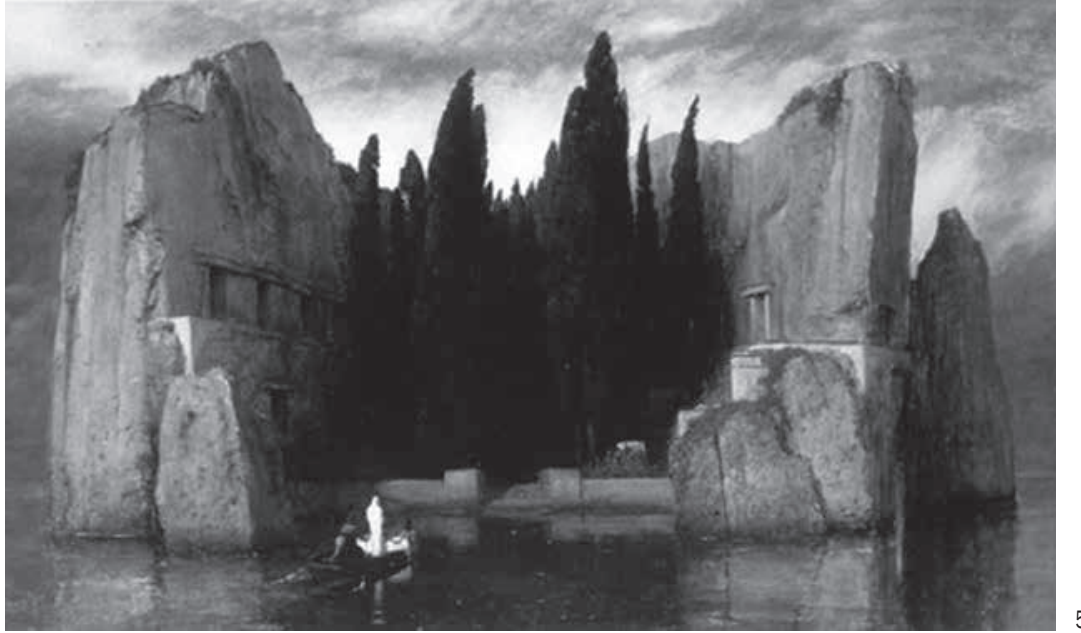

llegada de sus póstumos visitantes. Sólo el agua es capaz de rodear sus muros y de hacernos llegar hasta ella.

TRAZAS

'iResulta difícil imaginar los montones de cadáveres que durante siglos han acogido iglesias y claustros! Periódicamente, para hacer sitio, se quitaban del suelo de las igleslas y de los cementerios los huesos acabados de secar amontonándolos en las galerías de los osarios, en los forjados de las iglesias, debajo de los laterales de las bovedas, o se embutian en hoyos inutilizados, al lado de muros y pilares. De esta forma los visitantes de la iglesia, los clientes de las tiendas del cementerio, en efecto, los porches de cementerio con frecuencia se convertian en mercados, corrian sin cesar el riesgo de dar en algún resto huma caido de un osario u olvidado por un sepulturero"z.

El paso del tiempo ha conseguido reabsorber los antiguos camposantos de Venecia, como en la gran mayoría de las ciudades europeas. Cualduier ciudad es por si misma un cementerio que ha sido reabsorbido con absoluta naturalidad. La ciudad se convierte en un palimpsesto su huella componiendo un nuevo territorio. No debe sorprendernos que cuando paseamos por ellas es posible que estemos pisando aniguas necropolis o camposantos.

El edicto napoleónico que en 1804 ordenó que se alejatos camposantos del interior de Murano y Venecia consutiuye el punto de partida de la historia del futuro cementerio de San Michele. La primera isla que configuró el nuevo cementerio para los habitantes de la Laguna fue San Cristóforo della Pace. Inaugurado en 1813 y diseñado por Giovann Antonio Selva, pronto resultó insuficiente. Se amplio con la cercana ista de San Michele sin existir aún conexión física entre ellas (figura 4). Es en 1835 cuando se unen ambas islas, pasando a ser conocida como la isla de San Michele. En 1858 se convoca el concurso para el rediseño y la ampliación del cementerio del que resulto vencedor Annibale Forcellini, con una propuesta de marcado caracter ortogoposu
Resulta difíil imaginar lo que supuso en aquel tiempo el cierre y traslado de los camposantos. Desplazamientos, con todo el contenido poético que esto implica, que como barcos de Caronte, cruzaron la Laguna desde Venecia y Murano hasta San Michele (figura 5). Es irónico vensar que en la actualidad los difuntos, en su último
viajucen del mismo modo la Laguna a bordo de una barcaza o de una góndola, esa "singular embarcación tan típicamente negra como, entre todas las cosas de este mundo, lo son los ataúdes"s, hacia el cementerio de San Michele, como antaño lo hicieran los ciudadanos de la República Veneciana.

Muchas son las capas y estratos sedimentados en el Cementerio de San Michele, algunos ocultos, como la antigua isla de San Cristóforo della Pace, hoy irreconocible, otras se nos muestran visibles como la traza del cementerio original de 1835, la iglesia de San Michele de 1469 primer ejemplo de arquitectura renacentista de la ciuda y el claustro. Un continuo proceso de ampliaciones y adiciones respecto del proyecto inicial. Sobre la propuesta de Annibale Forcelli, que se prolongó durante más de un siglo debido a modificaciones en el proyecto original, se convoca en 1998 el concurso para la ampliación del cementerio de San Michele.

EL CONCURSO

Los concursos de arquitectura deberian funcionar como parentesis en la linea del tiempo. Espacios para la reflexion desde los que se lancen interrogantes, pausas en nuestro viaje en el que nos inundará la incertidumbre cuestiones que suscitarán nuevos encuentros y oportunidades capaces de generar ideas con un cierto grado de

2. Ariès, Philippe: El hombre ante la muerte. Madrid: Taurus, 1984 3. Mann, Thomas: La muerte en Venecia. Barcelona: Diario Público, 2010, libertad, herramientas que elaboren posibles soluciones sobre lugares revisiones del contexto temporal en el que se enmarcan as propuestas.

Las grandes necrópolis europeas sufrieron en su mayoría durante el siglo XX el problema de su propia saturación. En el caso de Venecia, las casi mil trescientas defunciones al año, sumado al lento proceso de mineralización de los cadáveres, dada la cercanía del nivel freático a las tumbas, imposibilitaban la renovación constante del camposanto. La colmatación del cementerio, y la necesidad de reutilización de los fangos generados por el saneamiento de los canales de la ciudad de Venecia fueron razones más que suficientes para esta nueva convocatoria Las posibles soluciones para afrontar esta ampliación pasaban por trasladar el nuevo cementerio, de nuevo, a otra isla de la Laguna o, como finalmente se decidió, ampliar cementerio existente.

Detenida en el tiempo, colmatada y clausurada, Venecia reúne como ninguna otra ciudad la imagen propia de un cementerio. Es paradójico pensar que mientras la ciudad se encuentra estancada, en el doble sentido, su cententerio sigue creciendo como si de una obra eterna se tratase. La ampliación se subdividia en dos fases con una supericie total de $60.000 \mathrm{~m}^{2}\left(15.000 \mathrm{~m}^{2}\right.$ correspondientes a la primera tase y $45.000 \mathrm{~m}^{2}$ de la segunda) Thua 6. La primera lase debía cerrar el perimetro rectangular de la isla de San Michele en su extremo nordeste, incorporando un atracadero y disponiendo la capilla, (t) unerarios. La segunda fase proponia la creación de una nueva isla en la zona este, junto a la existente, sobre la

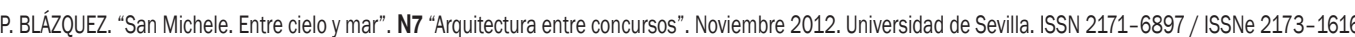




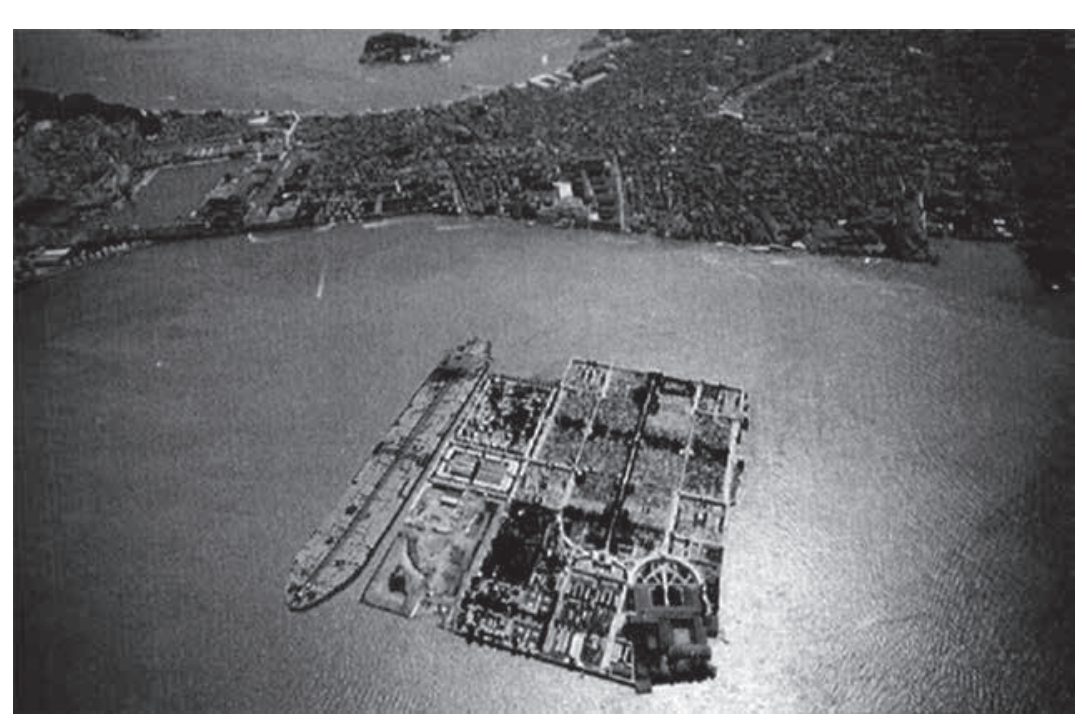

7. Fotomontaje de la propuesta. Carlos Fe rrater.

de la intervención. Carlos Ferrater 9. Alzado y sección longitudinal. Carlos Fe rrater.
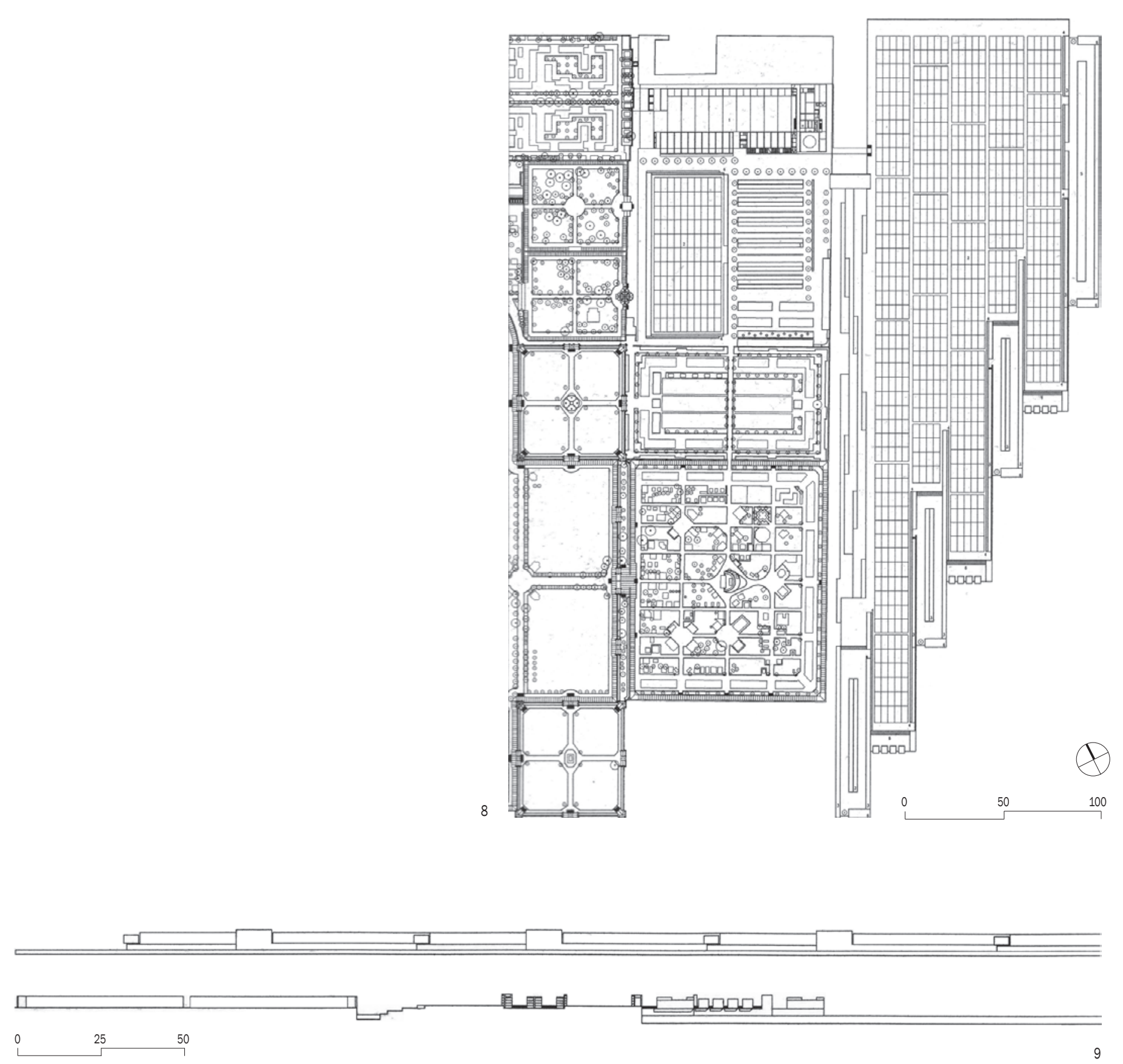

paisaje del cementerio, ligado a la horizontalidad de Laguna.

Nuestra cultura ha interpretado generalmente el cementerio como un recinto limitado, acotado y cerrado y quizás sea en este punto donde la propuesta de Carlos Ferrater se separe de las intenvenciones que plantean Enric Miralles y David Chipperfield. Cabe preguntarse si la respuesta a un camposanto acotado y colmatado como hasta ese momento lo era el de San Michele deba ser otro cementerio ensimismado.
ENRIC MIRALLES (SEGUNDO PREMIO) Miralles opta por una propuesta intrincada e incluso en algunos casos provocadora, abordando el concurso desde una estrategia completamente distinta al resto de las soluciones matando por completo la actuación en la isla de San Miche. tanto del proyecto, como del recorrido, y es un epílogo para 列 transición entre dos realidades diferentes y contrapuestas. Siviéndose de lafigura en espiral elaborada por Max Billy re e en claves orgánicas. La primera fase corresponde al inicio

ector venecianos, algo semejante a Aldo Rossi. El proyecto propone por un lado la primera ampliación con el límite ya establecido, por otro, el nuevo 


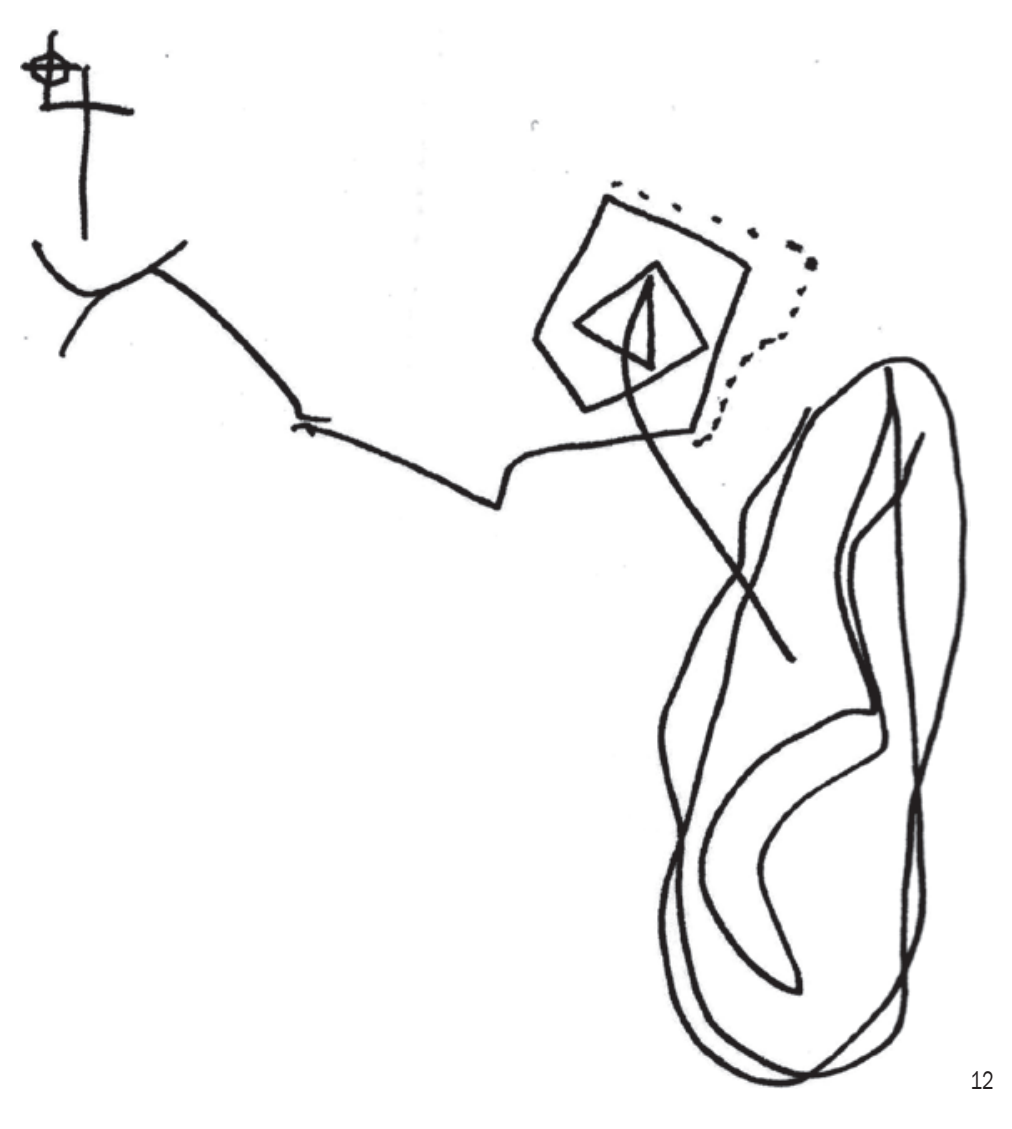

Si el tiempo es invisible, el proyecto de Miralles lo transforma en visible consiguiendo espacios que evocan lo inmaterial de un cementerio. Una propuesta entendida ano un proyecto inacabado, un juego de variaciones sada por gemación que el tiempo y la propia naturaba se encargarín de transformar para incorporarla al resdo de islas que componen el paisaje de la Laguna. En El tiempo, gran escultor, Marguerte Yourcenar escrib'a: "El día que una estatua está terminada, su vida en cieto sentido empieza. Se ha salvado a primera etapa que mediante los cuidados del escultor la ha llevado desde bloque hasta la forma humana una segunda etapa, en transcurso de los siglos, a través de alternativas de adoración, de admiración de amor de desprecio o de indiferencia por grados sucesivos de erosión y desgaste, la ír devolviendo poco a poco al estado mineral informe al que la había sustraído su escultor" ".

El croquis se convierte en uno de los elementos más sugerentes de la propuesta expresando gran parte de contenido de la propuesta (figura 12). Una vez completada la primera fase, se lanza una línea que nos lleva hacia la nueva isla, una tierra que en nada recuerda a la an-

reflexionar y disfrutar. El jardín que nunca ha tenido Venecia. Una nueva isla que como futuras ampliaciones de cementerio, podrían emerger de la Laguna. Es en el recorrido imaginado por Enric, "la promenade architectural" $d e$ la que hablara Le Corbusier, donde reside la potencia de su proyecto. Un recorrido cuyo centro está formado por lapilla y el crematorio, y con destino el mar. No es difíc imaginarnos en el antiguo camposanto de San Michele, rodeados por sus muros perimetrales, $y$ tomar el puente que nos conecta con la ampliación, la nueva isla emerg da, elevando nuestra mirada por encima de las tumbas, observando la inmensidad de la Laguna desde un nuevo punto de vista, a la altura de las cúpulas y los campanarios venecianos. "Ascender por el aire antes de volver a la .

DAVID CHIPPERFIELD (Primer Premio)

David Chipperfield obtuvo el primer premio del concurso introduciendo una variante distinta al resto de propuestas. La diferencia entre el proyecto del británico con respecto al de Miralles o Ferrater reside en la estructura organizativa que diseña, lo que le permite agrupar todos los usos del cementerio en una serie de piezas que construyen la cerrada por una cubierta ajardinada que reconstruirá el nivel del terreno y que gracias a las copas de los árboles terminarán por absorber la obra La reflexín en torno a tiempo resuena tras los proyectos de loualada y San Michele, ejerciendo un papel fundamental en la obra de Miralles, sobre todo después de leer las palabras del propio constantemente el concepto este caso se opta por cerrar la segunda isla después de haber provocado experiencies, recorridos y miradas que contecen el sentido final de aislamionto (figura 11) “ "Una tierra exhausta de este constante enterrar $y$ desenterrar entervary desenterar ${ }^{5}$, es la principal ráćn por la que tarquito abora la segunda fase creanch por la que isla en la Laguna, construyendo un nuevo territorio.

4. AA.WV.: Enric Miralles 1972-2000. Barcelona.: Fundacion Caja de Arquitectos, 2011

1995-2000. El Croquis. № 72. Madrid: El Croquis. 2000

6. Yourcenar, Marguerite: El tiempo, gran escultor. Madrid: Affaguara, 1989,

. 
de los cementerios que en el siglo XIX fueron trasladados a las afueras de las ciudades por cuestiones higiénicas como el de San Michele, se han vuelto a colmatar siendo reabsorbidos muchos de ellos por la trama urbana. Nuestra cultura ha interpretado este tipo arquitectónico como espacios que separan el interior del camposanto del exterior, excluyendolo de ningún tipo de relación con el ámbito que lo rodea. El concurso para la ampliación de San Michele es un excelente campo de pruebas desde el que poder analizar el contexto historico en torno a la ipología del cementerio y su relación con la ciudad y el erritorio. Una historia bajo la cual se visiumbran aspecos de proyecto, desplazamiento, reabsorción, reciclaje, ciudad, limite y territorio. Este texto intenta ser una de as posibles lineas de investigación desde la cual poder abordar el tema desde lo particular, una situacion real y concreta que reune alguna de las claves que nos servirán como cartas de presentacion para escribir las posibles hipotesis sobre las que tendremos que reflexionar.

A lo largo del articulo nos hemos desizado en la fronera entre el mundo de los vivos, y el de los muertos. ¿Es posible una revisión que establezca nuevas relaciones entre cementerio, ciudad y territorio? La crisis de los cementerios contemporáneos son el testimonio de la inquietud de nuestro tiempo, en el que han cambiando no sólo las prácticas sino tambien las creencias, mentalidades, normativas mortuorias, nitos funerarios como el de la incineración (prohibida por la Iglesia catolica hasta 1964 y en constante crecimiento) y representaciones colectivas que dibujan un nuevo escenario en torno a la figura de los cementerios. Transformaciones fundamentales que pueden suponer la desaparición de la tumba y el cementerio tal y como lo conocemos. Es posible entonces pensar que muchos de eslos camposantos comiencen a vaciarse dentro de 50 años, dando paso a un futuro proceso de desmantelamiento de cementerios hasta ahora inimaginable. Como si fuésemos arqueólogos del futuro, los actuales cementerios podrían convertirse en nuevas ruinas dentro de unos años, volviendo entonces a abandonar estos espacios en el interior de desplazamientos y reabsorciones en el espacio y el tiempo.
Toda creación es temporal y efímera, pero aspira desde un primer momento a durar eternamente. Sin embargo, el estado continuo de mutación al que se ven sometidas las sociedades ha provocado un proceso a lo largo del tiempo que ha alterado la relación de los cementerios con las ciudades y el territorio. ¿Cuál es la utilización que ha de tener en el mundo contemporáneo, en un sentido pragmático, operativo y productivo un cementerio? ¿Debe ser un cementerio una obra eterna? Podríamos plantearnos entonces si constatadas estas transformaciones, las propuestas que presentaron Carlos Ferrater y David Chipperfield en el año 1998 tendrían el mismo valor para el siglo XXI, o sí por el contrario el jurado se decantaría por una propuesta más acorde con la realidad actual. Quizás el jurado prestase más atención hoy a intervenciones más cercanas a la propuesta de Enric Mialles, que prevean la agotabilidad del propio cementerio pero que al mismo tiempo conviertan a la obra en eterna consiguiendo espacios que evolucionen y se transformen a través del tiempo, en la búsqueda de que conceptos como espacio y tiempo configuren el razonamiento de las propuestas.

La lectura que este texto pretende aportar expone que el concurso para la ampliación del cementerio de San Michele denota un nuevo punto de inflexion en la relaciones que se han establecido hasta ahora entre los cementelos, la ciudad y el teritorio. Una historia que debe ser observada en claves de desplazamientos y reabsorciones ciclicos que vuelven a situarnos en el principio de a invesigación, delatando la crisis de los cementerios, incapaces de encontrar un marco en el que establecer (onexiones produclivas y operalivas con la realidad conemporánea. Sólo una mirada hacia el futuro garantiza e abandono de antiguo usos, eficaces en su tiempo, pero a cora obsoletos. La uludad fijach en el tiempo conduce a un cementerio igualmente estático. Es necesario que los nuevos espacios para la muerte tengan una relación con "iudad mucho mas rica y mucho mas "viva".

"Hay dos unicas cosas imposibles de representar, el tresente y la muerte, la unica forma de llegar a ellas es a través de su ausencia"10.

\section{Bibliografía}

A..W.: Carlos Ferrater. Works and Projects 1980-2000. Barcelona: Actar, 1998

A.W.: Carlo Scarpa, 1906-1978. Milán: Electa, 2006.

A..W.: Cementerios de Andalucía. Sevilla: Junta de Andalucía Consejería de Obras Públicas y Transportes, 1993

AA.W.: Concurso $2 \mathrm{G}$ competition: Parque de la Laguna de Venecia = Venice Laggon Park. Barcelona: Gustavo Gili, 2008

AA.W.: Enric Miralles 1972-2000. Barcelona: Fundación Caja de Arquitectos, 2011

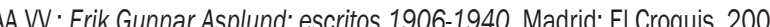

AA.W.: Forma y memoria. Barcelona: UPC, 2002

A.W. U: Una arquitectura para la muerte: actas del I Encuentro Internacional sobre los Cementerios Contemporáneos, Sevilla, 4.7 junio 1991. Sevilla

Ariès, Philippe: El hombre ante la muerte. Madrid: Taurus, 1984

Calvino, Italo: Las ciudades invisibles. Madrid: Siruela, 2007.

David Chipperfield 1991-2006. El Croquis. No 87. Madrid: El Croquis. 2006

Enric Miralles 1983-2000. El Croquis. № 34. Madrid: El Croquis. 2000

Enric Miralles + Benedetta Taǵliabue: 1995-2000. El Croquis. № 72. Madrid: El Croquis. 2000

Felicori, Mauro: Gli Spazi della memoria: architettura dei cimiteri monumentali europe. Roma: Luca Sossella, 2005

Martínez, Ángel: Sueños y Polvo: cuentos de tiempo sobre arte y arquitectura. Madrid: Lampreave, 2009,

Gili, Mónica: La última casa. Barcelona: Gustavo Gili, 1999

Giuffrè, María: Làrchitettura della memoria in Italia. Milán: Skia, 2007.

Loos, Adolf: Ornamento y delito y otros escritos. Barcelona. Gustavo Gili, 1972.

Mann, Thomas: La muerte en Venecia. Barcelona: Diario Público, 2010.

Muñoz, Juan: Juan Muñoz: Writingśs = Escritos. Madrid: La Central, 2009.

Dbras singulares. Arquitectura Viva. № 62 . Madrid: Arquitectura Viva. 1998.

Martí, Carlos; Portela, Cesar: Cementerio municipal en Fisterra, A Coruña_ 1977-1999. Almería: Colegio de Arquitectos de Almería, 2010.

Matvejevic, Predrag: La otra Venecia. Valencia: Pre-Textos, 2004.

Rodríguez Barberân, Francisco Javier; Ramos Guerra, Manuel: Cementerios de Andalucía: arquitectura y urbanismo. Sevilla: Consejería de Obra Públicas y Transportes, 1993.

Rossi, Aldo: Autobiografifia cientifica: Aldo Rossi. Barcelona: Gustavo Gili, 1998.

Rovira, Josep: Bohigas Oriol; Fundación Caja de Arquitectos" Enric Miralles: 1972-2000. Barcelona: Fundación Caja de Arquitectos, 2011.

Pablo Blázquez Jesús, Sevilla 1985. Arquitecto por la E.T.S.A. de Sevilla (2010). Socio-fundador de BSO arquitectura (WW

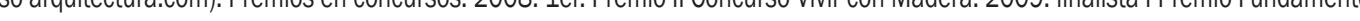
idad de Sevilla: 2011/12: Máster en Ciudad y Arquitectura Sostenible (E.T.S.A. de Sevillta. 


\section{Autor imagen y fuente bibliográfica de procedencia}

Información facilitada por los autores de los artículos: página 20, 1 (AA.W.: Concurso de Proyectos 1996. Propuestas de los equipos adjudicatarios. Sevilla: Dirección General de Arquitectura y Vivienda, Junta Andalucía, 1997, portada), 2 (Dirección General de Arquitectura y Vivienda, Junta Andalucía, tríptico); página 22, 3 (Manuel Toledo), 4 (Andrés López); página 23, 5 y 6 (Jesús Granada) 24, 7 (Fernando Alada), 8 (Jesús Granada), 9 (Fernando Alda); páginas 42, 43 y 44, 1 a 3 (El Croquis n53 0MA/Rem Koolhaas. Madrid: El Croquis, 1994, pp. 73 y 78); páginas 46, 47, 4 a 6 (AA.VV: Exposición Universal Sevilla 1992: ideas para una ordenación del recinto, Sevilla: Expo 92, Comisaría General de España, 1986); página 49, 7 (Ceccarelli, P. La construcción de la ciudad soviética. Barcelona: Gustavo Gili, S.A., 1972, figura 26, Anexo ilustraciones, p. XI), 8 (De Michelis, M.; Pasini, E. La Citta`Sovietica 1925-1937, Venecia: Marsilio Editori, 1976, p. 80); páginas 50y 51, 9 y 10 (9. De Michelis, M.; Pasini, E. La Citta 'Sovietica 1925-1937, Venecia: Marsilio Editori, 1976, pp. 81y 180); página 55,1 (Reproducido de Deutches Dokumentation fur Kunstgeschichte, Universitat Marburg, en www.fotomarburg.de); página 56, 2 (Cristina Gastón Guirao); página 57, 3 (Der Scherei nach den Turmhaus. Der IdeenwettbewerbHochhaus am Bahnhof Friedrichstrasse. Berlin 1921/22. Berlín: Argon Verlag GmbH, 1988, p. 39), 4 (Cristina Gastón Guirao en base a Der Scherei nach den Turmhaus. Der IdeenwettbewerbHochhaus am Bahnhof Friedrichstrasse. Berlin 1921/22. Berlín: Argon Verlag GmbH, 1988, p. 38); página 58, 5 (AAVV. Mies in Berlin. (publicado en relación con la exposición del mismo nombre). New York/Berlín: MOMA, 2001, p. 183); página 59, 6 (Reuter, Helmut; Schulte, Birgit. Mies and modern living. Interiors, furniture y photography. Ostfildern: Hatje Canz Verlag, 2008, p. 50, 210 y AAVV. Mies in Berlin. (publicado en relación con la exposición del mismo nombre). New York/Berlín: MOMA, 2001, p.181), 7 (AAVV. Mies in Berlin. (publicado en relación con la exposición del mismo nombre). New York/Berlín: MOMA, 2001, p. 182); página 60, 8 (Cristina Gastón Guirao en base a Der Scherei nach den Turmhaus. Der IdeenwettbewerbHochhaus am Bahnhof Friedrichstrasse. Berlin 1921/22. Berlin: Argon Verlag GmbH, 1988, pp. 45, 105); página 62, 9 y 10 (Reuter, Helmut; Schulte, Birgit. Mies and modern living. Interiors, furniture y photography. Ostfildern: Hatje Canz Verlag, 2008, pp. 230, 252, 258); página 64, 11 (Zevi, Bruno (ed): Erich Mendelsohn. Opera Completa. Turín: Testo \& Immagine, 1997, p. 197), 12 (Der Scherei nach den Turmhaus. Der IdeenwettbewerbHochhaus am Bahnhof Friedrichstrasse. Berlin 1921/22. Berlín: Argon Verlag GmbH, 1988, pp. 181, 184); página 65, 13 (Schulze, Franz. Mies van der Rohe. Una biografía crítica. Madrid: Hermann Blume, 1986 p. 155); página 69, 1 (Inv. Nr. HP 041,005. Architekturmuseum der Technischen Universität Berlin in der Universitätsbibliothek); páginas 70, 72, 73, 74, 2 a 5 (@FLC/Vegap, Sevilla, 2012. @Pierre Jeanneret, Vegap, Sevilla, 2012), 6 (Theaterwissenschaftliche Sammlung Universität zu Köln); página 75, 7 y 8 (Inv. Nr. 2750, Inv. Nr. F 1604. Architekturmuseum der Technischen Universität Berlin in der Universitätsbibliothek); páginas 76 y 80, 9 a 11 (@FLC/Vegap, Sevilla, 2012. @Alexander Rodchenko, Vegap, Sevilla, 2012), 12 (0148126. Tretyakov State Gallery, Moscow); página 82, 13 (Austrian Frederick and Lillian Kiesler Private Foundation Vienna), 14 ((СFLC/Vegap, Sevilla, 2012. @Pierre Jeanneret, Vegap, Sevilla, 2012); página 84, 15 (@FLC/Vegap, Sevilla, 2012. @Pierre Jeanneret, Vegap, Sevilla, 2012), 16 (X1119.3a-c. Colletion of Brooklyn Museum, New York); página, 85, 17 (Cabinet Ejzenstejn, Moscow (Hayuнoмемориальный кабинет-музей С. М. Эйзенштейна), 18 (Imagen de dominio público. http://commons.wikimedia.org/wiki/File:Sadanji_Ichikawa_Il_and_Sergei_Eisenstein. jpg); páginas 92 y 94, 1 y 2 (Roth, Alfred: La nouvelle architecture presentée en 20 exemples, Die neue architektur dargestellt an 20 Beispielen, The new architecture presented in 20 examples. 1930-1940. Zúrich: Verlag für Architektur Artemis, 1975, pp. 131, 132); página 94, 3 (Architettura, № XVI, Fasc. IX. Milán: septiembre de 1938, p. 574); página 96, 4 (Roth, Alfred: La nouvelle architecture presentée en 20 exemples, Die neue architektur dargestellt an 20 Beispielen, The new architecture presented in 20 examples. 1930-1940. Zúrich: Verlag für Architektur Artemis, 1975, p. 136), 5 (Fondazione Giuseppe Terragni; reproducida en Curtis, William J. R.: La arquitectura moderna desde 1900. Londres: Phaidon, 2006, p. 366); página 97, 6 (International Institute of Social History, Amsterdam; reproducida en Molema, Jan: Jan Duiker. Barcelona: Gustavo Gili, 1996, p. 85.), 7 (Emilio Cachorro Fernández); página 98, 8 y 10 (Roth, Alfred: La nouvelle architecture presentée en 20 exemples, Die neue architektur dargestellt an 20 Beispielen, The new architecture presented in 20 examples. 1930-1940. Zúrich: Verlag für Architektur Artemis, 1975, p. 137), 9 (AA.W.: Iǵnazio Gardella, 1905-1999. Arquitectura a través de un siǵlo. Catálogo de la exposición. Madrid: Ministerio de Fomento, 1999, p. 13); página 100, 11 y 12; página 102, 13 (Maffioletti, Serena: BBPR arquitectura. Banfi, Belgiojoso, Peressutti, Rogers. Sevilla: COAAO, 1996, pp. 47, 56, 67), 14 (Musée du Petit Palais, Ginebra; reproducida en Stoichita, Victor l.: Ver y no ver. Madrid: Siruela, 2005, p. 38); página 108, 1 (Arquitectura. Agosto 1969, № 128. Madrid: Colegio Oficial de Arquitectos de Madrid, 1969. Primera página; Nueva forma: arquitectura, urbanismo, diseño, ambientes, arte. "Concurso Universidad Autónoma de Madrid". Septiembre 1969. № 44. Madrid, 1969. Portada; Nueva forma: arquitectura, urbanismo, diseño, ambientes, arte. "Concurso Universidades Autónomas de Bilbao y Barcelona". Enero 1970. № 48. Madrid, 1970. Portada); página 110, 2 (Nueva forma. Septiembre 1969. № 44. cit. p. 5); página 111, 3 y 4 (Arquitectura. Agosto 1969, № 128. cit. pp. 33 y 35); páginas 112 y 113, 5 a 9 (Nueva forma. Septiembre 1969. № 44. cit. pp. 33, 30, 81, 18, 24); páginas 114, 115 y 116, 10 a 14 (Nueva forma. Enero 1970. № 48. cit. pp. 23, 20, 56, 69, 96), 15 y 16 (Cuadernos de arquitectura. Primer trimestre 1970. № 75. Barcelona: Colegio Oficial de Arquitectos de Catalunya, 1970. pp. 58 y 66); página 117, 17 (Nueva forma. Enero 1970. № 48. cit. p. 31); página 118, 18 (Nueva forma. Septiembre $1969 . N^{\circ} 44$. cit. p. 4; Temas de arquitectura y urbanismo. Septiembre 1969. № 123. Madrid-Barcelona, 1969. p. 5); página 123, 1 y 2 (Bucci, Ângelo; Puntoni, Álvaro; Vilela, José Oswaldo. Foto: Nelson Kon.); página 126, 3 (Parada, Sérgio Roberto. Projeto cedido por Rodrigo Biavati), 4 (Gorgati, Vinicius; Franco, Fernando de Mello; Moreira, Marta; Braga, Milton. Projeto cedido pelos autores), 5 (Rodrigues, Sidney Meleiros; Saraiva, Pedro Paulo de Melo; Rosemberg, Marcelo; Vaisman, Jacobina; Lobo, Marcos Toledo; Nunes, Ronaldo Soares.Projeto cedido pelos autores); páginas 128, 129, 6 a 12 (Bucci, Ângelo; Puntoni, Álvaro; Vilela, José Oswaldo. Projeto cedido pelos autores); página 131, 13 y 14 (Bucci, Ângelo; Puntoni, Álvaro; Vilela, José Oswaldo. Foto: Nelson Kon); página 136, 1 (Fromonot, Françoise. En Jørn Utzon, architetto della Sydney Opera House. Milano: Electa, Documenti di Architettura, 1998. pp. 15), 2 (Piano, Renzo. En Giornale di Bordo. Florencia: Passigli Editori, 1997, pag 38); página 138, 3 (Mikami, Yuzo. En Utzon's sphere. Sydney Opera House. How it was designed and built.Tokio: Ed. Shoku Kusha, 2001), 4 (Silver, Nathan, The making of Beuabourg, A building biography of the Centre Pomidou, Paris. Cambridge, Massachussets: Ed The MIT press, 1994); página 139, 5 (Piano, Renzo, Renzo Piano Building Workshop, complete works. Londres: Phaidon Press Limited, 1993. vol 1. pp. 54), 6 (Weston, Richard: Utzon : inspiration, vision, architecture. Hellerup (Dinamarca): Editorial Blondal, , 2002. pp. 117); página 140, 7 (Weston, Richard: Utzon: inspiration, vision, architecture. Hellerup (Dinamarca): Editorial Blondal, , 2002. pp 116), 8 (Fromonot, Françoise. En Jørn Utzon, architetto della Sydney Opera House. Milano: Electa, Documenti di Architettura, 1998. pp 64), 9 (Ferrer, Jaime, Jørn Utzon. Obras y proyectos. Barcelona: Editorial. Gustavo Gili, 2008. pp. 159); página 10, (Rice, Peter: "La structure métallique". En Architecture d'aujourd'hui. Febrero 1982, n 219. pp. 62. París: Editorial Groupe Expansion,1930), 11 (Piano, Renzo, Renzo Piano Building Workshop, complete works. Londres: Phaidon Press Limited, 1993. vol 1. pp. 45); página 142, 12 (Architectural Design', vol 47 nº 2, febrero 1977); página 144, 13 (Weston, Richard: Utzon : inspiration, vision, architecture. Hellerup (Dinamarca): Editorial Blondal, 2002); página 148, 1 (AA.W.: Arquitectura Viva. "Obras singulares". 1998, № 62. Madrid: Arquitectura Viva. 1998. pp. 78-81); página 149, 2 y 3 (AA.W.: Concurso 2G competition: Parque de la Laguna de Venecia = Venice Laggon Park. Barcelona: Gustavo Gill, 2008. pp. 81, 13); página 150, 4 (Jacopo de 'Barbari, [Web en linea]. [Consulta: 04-10-2012]), 5 (Zumthor, Peter: Atmósferas: entornos arquitectónicos, las cosas a mi alrededor. Barcelona: Gustavo Gili, 2006. p. 6); página 151, 6 (David Chipperfield 2006-2009. El Croquis. № 120. Madrid: El Croquis. 2008. p. 66); páginas 152, 153,7 a 9 (AA.W.: Carlos Ferrater. Works and Projects 1980-2000. Barcelona: Actar, 1998); páginas 154, 155, 10 a 12 (Enric Miralles + Benedetta Tagliabue: 1995-2000. El Croquis. № 72. Madrid: El Croquis. 2000. pp. 132-141); página 156, 13 a 15 (David Chipperfield 2006-2009. El Croquis. № 120. Madrid: El Croquis. 2008. pp. 64, 67, 66); página 157, 16,17 (Luca Nicolao Photography, [Web en linea]. [Consulta: 19-9-2012]), 18 (David Chipperfield 2006-2009. El Croquis. № 120. Madrid: El Croquis. 2006. p. 66); páginas 162, 164, 167, 169, 170, 1 a 6 (๑ F.L.C. /VEGAP, Sevilla, 2012. OPierre Jeanneret, VEGAP, Sevilla, 2012); página 176, 1 (García Mercadal y S. Giedion. Congresos internacionales de arquitectura moderna. AC. №5. Primer Trimestre de 1935. Barcelona. GATEPAC.1932 p.12); páginas 178, 179, 2 a 4 (AC. №11. Tercer trimestre de 1933. Barcelona. GATEPAC. 1933. pp. 37, 34, 35); página 180, 5 (Le Corbusier et Pierre Jeanneret : Ouvre Complète 1910-1929. Zurich : Les Édtions D’Architecture. 1964 p.65), 6 (AC. NN11. Tercer trimestre de 1933. Barcelona. GATEPAC. 1933. p. 34), 7 (Le Corbusier et Pierre Jeanneret: Ouvre Complète 1910-1929. Zurich : Les Édtions D’Architecture. 1964 p.65), 8 (L'Habitation minimun. Frankfurt. 1929. Zaragoza: Edición facsimil Colegio de Arquitectos de Aragón.1997.p.26), páginas 181, 182 (AC. №11. Tercer trimestre de 1933. Barcelona. GATEPAC. 1933. p. 37, 1); páginas 189, 190, 1 y 2 (Cássia de Souza Mota); página 191, 3 (Boero, Dolores; Castellote, Ana Maria; Puglisi, Jose Agustin. Fonte: http://www.uia-architectes.org/texte/ england/Celebration/SPrix/Reglll/Frames.html), 4 (Fernandez, Alberto; Guerrero, Camilo; Javiera, Paulina; Sanchez Recio, José Manuel. Fonte: http://www.celebcities2.org/); página 192, 5 y 6 (Nikita, Sergienko. Fonte: http://www.celebcities3.org/wc/content/18/pdf/054FECC2-C9DC-410A-A044-CB2A0BBD25EB.pdf) 